\title{
Complex Cytokine Responses in Imported Scrub Typhus Cases, Germany, 2010-2018
}

\author{
Philip Eisermann, ${ }^{1 *} \dagger$ Jessica Rauch, ${ }^{1} \dagger$ Stefan Reuter, ${ }^{2}$ Lukas Eberwein, ${ }^{2}$ Ute Mehlhoop, ${ }^{1}$ Petra Allartz, ${ }^{1}$ Birgit Muntau, ${ }^{1}$ \\ and Dennis Tappe ${ }^{1}$ \\ ${ }^{1}$ National Reference Center for Tropical Pathogens, Bernhard Nocht Institute for Tropical Medicine, Hamburg, Germany; ${ }^{2} 4$ th Department of \\ Internal Medicine, Klinikum Leverkusen gGmbH, Leverkusen, Germany
}

\begin{abstract}
Scrub typhus is a life-threatening zoonotic disease, which is caused by Orientia tsutsugamushi, an obligatory intracellular Gram-negative bacterium. It is transmitted by Leptotrombidium mites in endemic regions of Southeast Asia. So far, data on imported scrub typhus cases to non-endemic areas and immunological descriptions are rare. Eleven scrub typhus cases that had been diagnosed by the German National Reference Center for Tropical Pathogens between 2010 and 2018 were retrospectively reviewed for clinical symptoms, laboratory changes, and travel destinations. Patient sera were included if follow-up samples showed simultaneous seroconversion for lgM and IgG antibody responses by immunofluorescence assays or concurrence with the first serum sample. The median of seroconversion was week 2 after symptom onset. Cytokine levels were measured over time, demonstrating simultaneously upregulated major Th1, Th2, and Th17 cytokines in the acute phase of infection followed by normalization during convalescence. This study underlines the complex mixed cytokine response elicited by scrub typhus and highlights clinical and diagnostic aspects of imported infections with O. tsutsugamushi.
\end{abstract}

\section{INTRODUCTION}

Scrub typhus is a vector-borne infection caused by Orientia tsutsugamushi. The obligate intracellular bacterium is transmitted by bites of larval stage Leptotrombidium mites. The inoculation of the bacteria causes a local inflammatory reaction often resulting in a necrotic lesion, the eschar. Systemic manifestations occur 5-14 days after infection, comprising most frequently fever, a maculopapular rash, lymphadenopathy, headache, and myalgia. Severe complications such as myocarditis, acute renal failure, pneumonia, and meningoencephalitis might develop, which can lead to multiple organ failure with a high case fatality rate if not adequately treated. Scrub typhus is endemic in a triangular geographic region between eastern Russia, Japan, Pakistan, and Australia. ${ }^{1}$ However, confirmed autochthonous Orientia sp. infections have been recently described in $\mathrm{Chile}^{2}$ and the United Arab Emirates. ${ }^{3}$ Furthermore, there are also reports about possible scrub typhus cases in East Africa, ${ }^{4,5}$ underlining the emerging nature of this disease. Little is known about the immunology of human infection with $O$. tsutsugamushi, however.

In this study, we retrospectively analyzed imported scrub typhus cases that had been diagnosed at the National Reference Center for Tropical Pathogens between 2012 and 2018. Clinical data were collected, and cases were serologically analyzed. Antibody kinetics was determined from followup sera. Serum cytokine responses were measured by flow cytometry from all available sera.

\section{MATERIALS AND METHODS}

Ethics statement. All data analyzed were anonymized. Informed consent was obtained from all individual participants included in the study.

\footnotetext{
*Address correspondence to Philip Eisermann, National Reference Center for Tropical Pathogens, Bernhard Nocht Institute for Tropical Medicine, Bernhard-Nocht-Str. 74, Hamburg 20359, Germany. E-mail: eisermann@bnitm.de

†These authors contributed equally to this work.
}

Cases and inclusion criteria. The database of the German Reference Center for Tropical Pathogens at the Bernhard Nocht Institute for Tropical Medicine, Hamburg, was screened for imported scrub typhus cases diagnosed from January 1, 2010 to December 31, 2018. Scrub typhus cases were defined as a clinically compatible disease with at least one of the following laboratory test results: a positive polymerase chain reaction (PCR) testing, a seroconversion to $O$. tsutsugamushi antigens in an indirect immunofluorescence antibody test (IFAT), a parallel $\operatorname{lgM}$ and IgG detection against $O$. tsutsugamushi antigens in a single sample using IFAT, or a single IFAT IgG or total Ig titer of $\geq 1: 320$. In addition, serology for typhus group and spotted fever group rickettsiae (in-house IFATs), leptospirosis (in-house ELISA), and dengue fever (in-house IFAT) had to be negative.

Serological and molecular assays. In-house scrub typhus IFAT was performed using $O$. tsutsugamushi strain Karp in L929 mouse fibroblast cell culture. Immunofluorescence antibody test reference values were $<1: 40(\operatorname{lgM})$ and $<1: 80(\operatorname{lgG}$ and total lg). All the reference values were determined with sera from 200 healthy Caucasian blood donors. Orientia tsutsugamushi-specific real-time quantitative PCR (qPCR) was performed targeting the $56 \mathrm{kDa}$ antigen gene. ${ }^{6}$ Depending on the clinical case and the available sample material, qPCRs were performed with DNA extracts from EDTA blood, cerebrospinal fluid, or eschar biopsy samples.

Cytokine measurements. Serum cytokine responses were analyzed by LEGENDplex (BioLegend, Fell, Germany) from all available sera. Thirteen sera from healthy blood donors served as controls. For cytokine analysis, blood sampling dates of the patients were assigned to the acute phase of infection (weeks 1-4 after symptom onset) and convalescent phase ( $>4$ weeks after symptom onset). The standard detection limits of the LEGENDplex assay for the analyzed cytokines were as follows: basic fibroblast growth factor $(24.2 \mathrm{pg} / \mathrm{mL})$, eotaxin $(6.9$ $\mathrm{pg} / \mathrm{mL}$ ), granulocyte colony-stimulating factor (G-CSF, 10.7 $\mathrm{pg} / \mathrm{mL}$ ), granulocyte-macrophage colony-stimulating factor (GM-CSF, $6.7 \mathrm{pg} / \mathrm{mL}$ ), interferon- $\alpha$ (IFNa, $1.2 \mathrm{pg} / \mathrm{mL}$ ), interferon- $\mathrm{Y}$ (IFNy, $5.2 \mathrm{pg} / \mathrm{mL})$, interleukin (IL) $1 B(4.1 \mathrm{pg} / \mathrm{mL})$, IL2 (4.3 pg/mL), IL-4 (4.8 pg/mL), IL-5 (4.0 pg/mL), IL-6 (4.4 pg/ $\mathrm{mL}), \mathrm{IL}-8$ (1.5 pg/mL), IL-9 (4.0 pg/mL), IL-10 (3.9 pg/mL), IL$12 \mathrm{p} 70$ (19.5 pg/mL), IL-13 (3.3 pg/mL), IL-17A (3.7 pg/mL), 
IL-17F (5.0 pg/mL), IL-21 (6.4 pg/mL), IL-22 (19.5 pg/mL), IFNy-induced protein 10 (IP-10, N/A), monocyte chemotactic protein-1 (MCP-1, N/A), macrophage inflammatory protein-1 $\alpha$

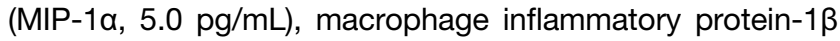
(MIP-1 $\beta, 5.2 \mathrm{pg} / \mathrm{mL}$ ), platelet-derived growth factor BB (PDGF-BB, N/A), regulated on activation, normal $T$ cell expressed and secreted (RANTES, $5.2 \mathrm{pg} / \mathrm{mL}$ ), tumor necrosis factor- $\alpha$ (TNF $\alpha, 4.4 \mathrm{pg} / \mathrm{mL}$ ), and vascular endothelial growth factor (VEGF, $26.9 \mathrm{pg} / \mathrm{mL}$ ).

Statistical analysis was performed with GraphPad Prism 7 (GraphPad Software Inc., La Jolla, CA). For comparison between the analyzed groups, the Kruskal-Wallis test and subsequent Dunn's multiple comparisons test were used.

\section{RESULTS}

A total of 11 scrub typhus patients were identified (Table 1), with an age range of $19-70$ years (mean age 42.5 years) and a male : female ratio of 2.7:1. Scrub typhus was acquired during travel in Southeast Asia (Thailand, Laos, Vietnam, South Korea, Malaysia, and Myanmar) and Nepal. The exposure to mites had not been specifically asked.

Most patients had fever on admission (10 patients, 91\%) followed by exanthema (6 patients, 55\%; Figure 1A), headache (5 patients, $45 \%$ ), eschar (5 patients, 45\%; Figure 1B), and myalgia/arthralgia (3 patients, $27 \%$ ). The most common combination of symptoms was fever and headache, which was seen in five patients (45\%). Lymphadenopathy and splenomegaly were found in two patients (18\%). Neurological signs, respiratory failure, and diarrhea were noted in one patient each. Hospitalization was necessary in a total of eight patients (73\%). Patients had received doxycycline treatment (200 mg/d for 5-21 days) in the country of travel or on return. After treatment, patient 5 showed arthralgia and mild cephaIgia, whereas patient 8 presented with an incomplete tetraparesis at the final clinical follow-up examination. All other patients recovered from the infection without sequelae.
Laboratory changes were found in the medical records of six patients on admission and included increased levels of C-reactive protein (CRP) and elevated liver enzymes in five patients (83\%). Anemia, increased concentrations of lactate dehydrogenase (LDH), and thrombocytopenia were seen in two patients (33\%), whereas eosinopenia was present in one patient $(17 \%)$. Of note, patients were observed at different days of illness in different hospitals.

Polymerase chain reaction was performed on clinical samples from three patients. A positive PCR result for O. tsutsugamushi was obtained in two cases, from an eschar biopsy and whole blood in patient 9 and from an eschar biopsy sample alone in patient 1 (Table 1). No genotyping of O. tsutsugamushi was performed.

Scrub typhus serology was performed in nine patients (82\%). From the two PCR-positive individuals, no serum was available. Antibodies against $O$. tsutsugamushi antigens were detected earliest in week 1 of illness, and the median of seroconversion was week 2 . Seroconversion (with parallel IgM and $\lg \mathrm{G}$ detection) was observed in three patients (33\%), whereas the presence of $\lg M$ and $\lg G$ in the first sample was seen in five patients (56\%). In one patient (patient 8), only lgM and total Ig were positive, with no specific IgG seroconversion after four weeks of illness.

Serum cytokines could be measured in nine patients, in two of them also at two different time points. Eight serum samples were assigned to the acute phase of illness and three samples to the convalescent phase of the infection. Almost all the measured serum cytokines and chemokines were significantly elevated in patients in the acute phase of illness in comparison with healthy controls (Figure 2). These include the serum levels of eotaxin, FGFb, G-CSF, GM CSF, IFNa, IFNy, IL-1ß, IL-2, IL-4, IL-5, IL-6, IL-8, IL-9, IL-10, IL-13, IL-17A, IL-17F, IL-21, IL-22, IP-10, MIP-1a, MIP-1B, TNFa, and VEGF. Although the concentrations of IFNa, IL-17F, and VEGF remained elevated in the convalescent phase of the infection, levels of all other chemokines/cytokines decreased again and were comparable

TABLE 1

Characteristics of 11 patients with imported scrub typhus, Germany, 2010-2018*

\begin{tabular}{|c|c|c|c|c|c|c|}
\hline Patient no. & Age, year/gender & Year of diagnosis & Travel history & PCR result $†$ & Signs and symptoms & Hospitalized \\
\hline 1 & $55 / \mathrm{M}$ & 2018 & Laos & Positiveł & Eschar, exanthema, fever, and headache & Yes \\
\hline 2 & $50 / F$ & 2018 & Laos and Thailand & ND & $\begin{array}{l}\text { Exanthema, fever, headache, and } \\
\text { splenomegaly }\end{array}$ & Yes \\
\hline 3 & $34 / \mathrm{M}$ & 2017 & Vietnam & ND & Exanthema, fever, and diarrhea & Yes \\
\hline 4 & $35 / \mathrm{M}$ & 2016 & Thailand and Malaysia & ND & $\begin{array}{l}\text { Exanthema, fever, severe headache, } \\
\text { lymphadenopathy, myalgia, and } \\
\text { arthralgia }\end{array}$ & No \\
\hline 5 & $70 / \mathrm{M}$ & 2015 & Nepal & ND & $\begin{array}{l}\text { Fever, headache, lymphadenopathy, } \\
\text { arthralgia, and splenomegaly; sequelae } \\
\text { on last follow-up examination }\end{array}$ & Yes \\
\hline 6 & 25/M & 2014 & South Korea & ND & Eschar and fever & No \\
\hline 7 & $27 / F$ & 2014 & Thailand & ND & Fever and severe headache & Yes \\
\hline 8 & $51 / \mathrm{M}$ & 2014 & Thailand & Negative§ & $\begin{array}{l}\text { Ataxia, bilateral facial paresis, diplopia, } \\
\text { ophthalmoplegia, tetraparesis, and } \\
\text { respiratory failure; sequelae on last } \\
\text { follow-up examination }\end{array}$ & Yes \\
\hline 9 & $61 / \mathrm{M}$ & 2014 & Myanmar & Positive & Eschar, exanthema, fever, and arthralgia & Yes \\
\hline 10 & 19/M & 2013 & Vietnam & ND & Eschar, exanthema, and fever & No \\
\hline 11 & $41 / \mathrm{F}$ & 2012 & South Korea & ND & Eschar and fever & Yes \\
\hline
\end{tabular}

$\mathrm{PCR}=$ polymerase chain reaction; $\mathrm{M}=$ male; $\mathrm{F}=$ female.

${ }^{*} \mathrm{ND}=$ not performed (whole blood or biopsy sample not available).

† Quantitative PCR targeting the $56 \mathrm{kDa}$ gene of Orientia tsutsugamushi.

$\ddagger$ Positive from eschar biopsy sample.

$\S$ Negative from cerebrospinal fluid.

\ Positive from eschar biopsy sample and whole blood. 


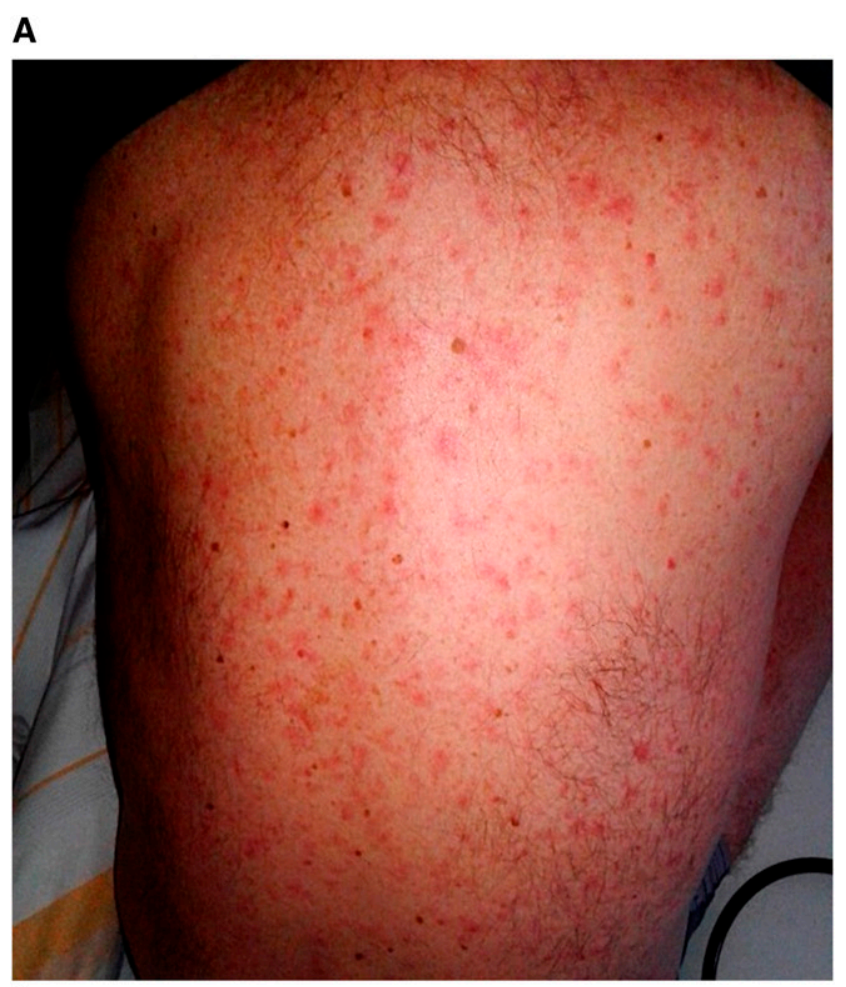

B

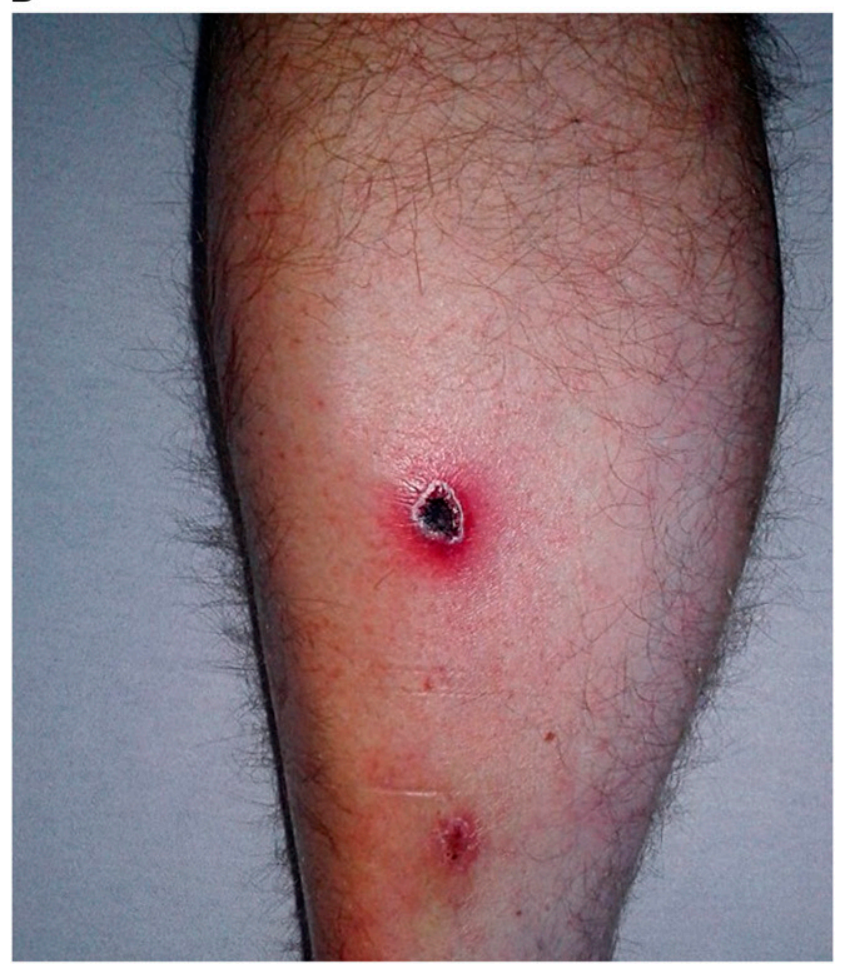

FIGURE 1. Typical exanthema and eschar in a scrub typhus patient (patient 1). (A) Maculopapular and non-pruritic rash on the back. (B) Eschar located on the shin of the same patient after travel to Laos.

with the concentrations of the control group (Figure 2). The levels of IL-12, PDGF BB, and MCP-1 were similar in both the acute and convalescent phases in patients and controls (data not shown). Of note, the IL-12 concentrations were only elevated in the serum of two patients in the acute phase of illness (patient 1, $226.7 \mathrm{pg} / \mathrm{mL}$ and patient 4, $130.4 \mathrm{pg} / \mathrm{mL}$ ). Interestingly, the levels of RANTES were found to be significantly reduced in the acute phase of illness compared with healthy controls. In the serum of one patient (patient 5), the concentrations of most cytokines and chemokines were markedly higher during the acute phase of infection than in the other patients.

\section{DISCUSSION}

Reports about imported scrub typhus cases to nonendemic regions are rare. According to a recent review of the available data, fewer than 40 patients have been reported. ${ }^{7}$ The overall majority of published data describe imported infections from the traditional area of the tsutsugamushi triangle. ${ }^{7-11}$ In line with these reports, all infections with $O$. tsutsugamushi in our study were acquired in countries located in Southeast Asia and in Nepal. In contrast to the few reported cases in travelers, at least one million cases of scrub typhus occur in the Asian-Pacific region annually. ${ }^{12}$ Insufficient clinical experience and unavailable diagnostic methods in many non-endemic regions might explain this lack of published data. Unarguably, scrub typhus is an important differential diagnosis in travelers returning from endemic regions presenting with an acute febrile illness. Furthermore, a report about an imported Orientia sp. infection from the Middle East to Australia ${ }^{3}$ and autochthonous scrub typhus cases in Chile ${ }^{2}$ should raise awareness of the emergence of this disease in unexpected regions.

Most patients in our study presented with nonspecific symptoms such as fever (91\%), exanthema (55\%), and headache $(45 \%)$. Prevalence of the characteristic eschar in scrub typhus patients can vary greatly ${ }^{13-15}$ and might depend on the O. tsutsugamushi genotype. ${ }^{16}$ In our study, nearly half of the patients presented with an eschar. In patient 8 , an eschar was not detected and treatment with doxycycline was delayed. This patient developed meningoencephalitis and an acute respiratory distress syndrome, leading to prolonged recovery with incomplete tetraparesis. The absence of an eschar seems to be associated with more severe symptoms and complications by hindering swift diagnosis and early commencement of adequate treatment. ${ }^{17,18}$ Meningoencephalitis is a well-documented complication of scrub typhus. The clinical outcome is generally benign, but chronic sequelae and fatalities might occur. ${ }^{19}$

Laboratory data were not available from all patients. Increased levels of CRP and liver enzymes were detected in more than $80 \%$ of patients, followed by anemia, thrombocytopenia, and elevated serum concentration of LDH. These results are in line with previous reports about imported scrub typhus cases. ${ }^{7,8}$ According to studies in endemic areas, laboratory changes are not as frequent as in imported cases, but more studies with larger numbers of imported $O$. tsutsugamushi infections are needed to draw further conclusions. ${ }^{20,21}$

Detection of antibodies against $O$. tsutsugamushi by IFAT is the gold standard in diagnosing scrub typhus. ${ }^{22}$ IgM seroconversion is usually detectable by the end of the first week after symptom onset, followed by IgG near the end of the second week. ${ }^{23}$ In our study, the earliest detection of antibodies was in the first week and the median time of seroconversion was week 2 . Of importance, serum from each patient had not been collected on the same day, so the data 

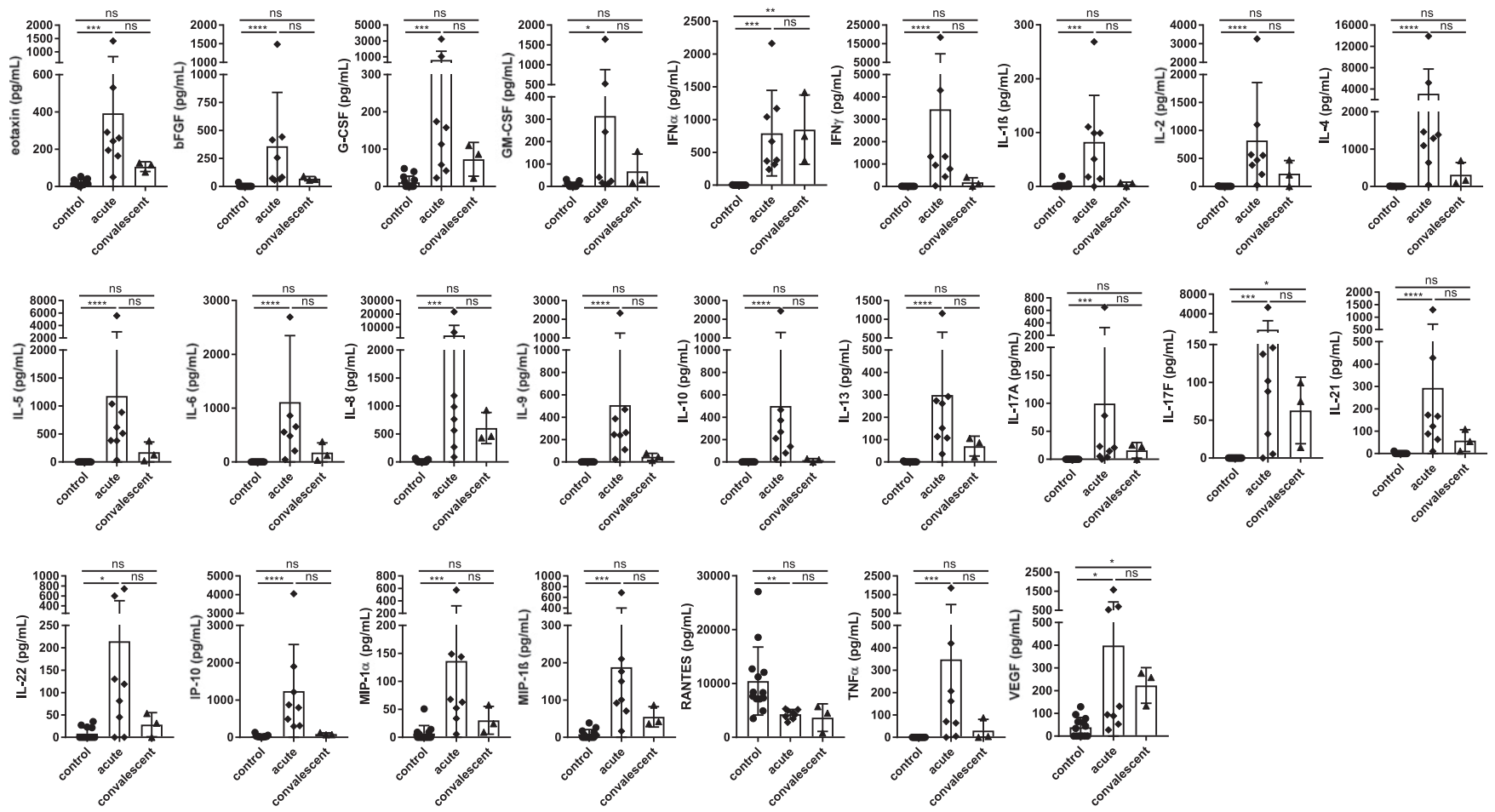

FIGURE 2. Cytokine and chemokine levels in imported scrub typhus cases. Eleven sera from nine patients with scrub typhus and 13 sera from healthy blood donors (controls) were analyzed in parallel by bead-based LEGENDplex assay. Eight serum samples were assigned to the acute phase of scrub typhus (weeks 1-4 after symptom onset) and three samples to the convalescent phase of the infection (> 4 weeks after symptom onset). Most serum cytokine and chemokine levels started to increase during the acute phase of illness and decreased again in the convalescent phase. Data are expressed as mean \pm SD. Statistical analyses were performed with the Kruskal-Wallis test and subsequent Dunn's multiple comparisons test. Asterisks indicate statistically significant differences $\left({ }^{\star} P<0.05\right.$, ${ }^{\star \star} P<0.01$, ${ }^{\star \star \star} P<0.001$, ${ }^{\star \star \star \star} P<0.0001$ ); ns $=$ not significant.

can only serve as estimates. In more than half of the individuals, IgG and IgM were already present in the first serum sample. In the remaining patients, IgM and IgG seroconverted simultaneously, except for patient 8 who did not show lgG seroconversion and who suffered from scrub typhusassociated meningoencephalitis. The reason for the lack of IgG seroconversion and whether this was a predisposition for the severe clinical course remain unsolved. Because of the delayed antibody response, clinical diagnosis can often only be confirmed in retrospect. ${ }^{24}$ Direct early pathogen detection before seroconversion by PCR might initiate immediate effective treatment. Here, eschar biopsy samples from two patients were analyzed by PCR, with positive results. Samples from the remaining three individuals, who presented with an eschar, were not available. Several studies have shown that PCR using eschar tissue is more sensitive than PCR with blood samples. ${ }^{23}$ We were also able to detect O. tsutsugamushi DNA in a whole blood sample during the acute phase of the disease before antibiotic treatment was started. However, after treatment is initiated, pathogen detection by PCR in blood samples usually is not possible, whereas eschar tissue PCR still can yield positive results for up to 7 days, enlarging the diagnostic window. ${ }^{25}$

Here, we report data on the systematic inflammatory response in scrub typhus patients in the acute and convalescent phases of illness. To our knowledge, this is the first study to measure a broad spectrum of cytokines and chemokines in a case series of imported scrub typhus. The investigation shows that 22 human cytokines and chemokines, including the major Th1, Th2 and Th17 cytokines, were simultaneously upregulated in the sera of patients in the acute phase and declined again after 4 weeks during convalescence. Why most cytokines and chemokines were markedly elevated in the serum from the acute phase of the elderly patient 5 is unclear. This patient had anemia, thrombocytopenia, and eosinopenia and showed elevated liver enzymes and increased CRP and $\mathrm{LDH}$ concentrations. He suffered later from relatively mild sequelae.

Significantly increased serum levels of IFNy and TNFa in the acute phase in combination with elevated IL-12 production, at least in two patients, are consistent with a Th1 immune pattern. The expression of IFNy seems to be protective against $O$. tsutsugamushi infection in mice, ${ }^{26-28}$ and our findings confirm previously published data that the IFNy concentration is elevated in the serum of scrub typhus patients during the acute phase. ${ }^{6,29}$ The role of TNFa is not as clear during the $O$. tsutsugamushi infection: On the one hand, TNFa can propagate a strong Th1 response, leading to elimination of the pathogen, but on the other hand, serum concentrations of TNFa correlate with the severity of scrub typhus, indicating a fatal role of TNFa in the pathogenesis. ${ }^{29}$ In addition, increased levels of cytokines such as IL-1 $\beta$, IL-4, IL-6, IL-13, IL-17, IL-21, and IL-22 in the sera of our patients underline a simultaneous Th2 and Th17 response, so Th1-, Th2-, and Th17-type responses do not seem to be clearly polarized. IL-1ß, IL-6, and IL-21 have been shown to initiate the differentiation of $T$ cells toward the Th17 lineage, and the main effector cytokines of Th17 cells are IL-17 and IL-22. ${ }^{30-32}$ Th17 cells became the focus of attention as they play a protective role during bacterial infections; however, they seem to mediate tissue 
damage. ${ }^{33-36}$ A correlation of pathologies such as hepatitis with high IL-17 levels in the serum of $O$. tsutsugamushiinfected patients could not be found by others, ${ }^{37}$ so it is likely that the Th17-type response is rather beneficial for the host than detrimental. In addition, a number of chemokines were found to be upregulated during the acute phase of infection, including IL-8, IP-10, MCP-1, MIP-1 $\alpha$, and MIP-1 $\beta$. These chemokines attract leukocytes such as neutrophils and macrophages into the inflamed tissue and promote leukocyte-endothelial cell interaction during inflammation. Interestingly, MIP-1 $\alpha$, MIP-1 $\beta$, and MCP-1 correlated with vascular inflammatory cell infiltration in in vivo studies in murine models of $O$. tsutsugamushi infection, ${ }^{27}$ and this could contribute to vascular permeability observed in patients with severe scrub typhus. By contrast, the expression of the chemokine RANTES was significantly reduced in the acute and convalescent phases in our patients, which has also been detected in scrub typhus patients before, associated with disease severity and fatal outcome. ${ }^{38}$

However, not only pro-inflammatory mechanisms were initiated during the acute phase of infection but also antiinflammatory responses. Th2-derived cytokines such as IL-4, IL-5, IL-6, and IL-13 are not just essential for B cell differentiation and isotype switching, but IL-4 is also described to limit or attenuate tissue damage due to its anti-inflammatory properties, which include the suppression of pro-inflammatory and Th1-type responses. ${ }^{26,39,40}$ In addition, the observed high expression of the anti-inflammatory cytokine IL-10 in the acute phase of infection in our patients and also other studies addressing scrub typhus represents an additional counterbalancing mechanism to ensure homeostasis within the host. ${ }^{6,29,38,41}$ Its antagonistic effect against further pro-inflammatory cytokine production, also confirmed by the detection of the diminished cytokine production in the convalescent phase in our patients, exerts an inhibitory mechanism on the immune response and potentially prevents further pathological tissue alterations. Of note, these mechanisms can lead to diminished clearance of the pathogen, but they could also prevent overshooting immune reactions and, thus, reduce damage during clearance of the pathogen.

In conclusion, our study shows a mixed cytokine pattern in acute scrub typhus cases. More studies, including $\mathrm{T}$ cell response analyses, are needed to shed more light on the immunology and pathophysiology of scrub typhus. In addition, the presented clinical and diagnostic data may guide clinicians in non-endemic countries toward a swift diagnosis and the initiation of early effective treatment procedures.

Received July 2, 2019. Accepted for publication October 9, 2019.

Published online November 25, 2019.

Authors' addresses: Philip Eisermann, Jessica Rauch, Ute Mehlhoop, Petra Allartz, Birgit Muntau, and Dennis Tappe, National Reference Center for Tropical Pathogens, Bernhard Nocht Institute for Tropical Medicine, Hamburg, Germany, E-mails: eisermann@bnitm.de, rauch@bnitm.de, mehlhoop@bnitm.de, allartz@bnitm.de, muntau@ bnitm.de, and tappe@bnitm.de. Stefan Reuter and Lukas Eberwein, 4th Department of Internal Medicine, Klinikum Leverkusen $\mathrm{gGmbH}$, Leverkusen, Germany, E-mails: stefan.reuter@klinikum-lev.de and lukas.eberwein@klinikum-lev.de.

This is an open-access article distributed under the terms of the Creative Commons Attribution License, which permits unrestricted use, distribution, and reproduction in any medium, provided the original author and source are credited.

\section{REFERENCES}

1. Xu G, Walker DH, Jupiter D, Melby PC, Arcari CM, 2017. A review of the global epidemiology of scrub typhus. PLoS Negl Trop Dis 11: e0006062.

2. Weitzel T, Dittrich S, Lopez J, Phuklia W, Martinez-Valdebenito C, Velasquez K, Blacksell SD, Paris DH, Abarca K, 2016. Endemic scrub typhus in South America. N Engl J Med 375: 954-961.

3. Izzard L et al., 2010. Isolation of a novel Orientia species (O. chuto sp. nov.) from a patient infected in Dubai. J Clin Microbiol 48: 4404-4409.

4. Horton KC, Jiang J, Maina A, Dueger E, Zayed A, Ahmed AA, Pimentel G, Richards AL, 2016. Evidence of Rickettsia and Orientia infections among abattoir workers in Djibouti. Am J Trop Med Hyg 95: 462-465.

5. Maina AN, Farris CM, Odhiambo A, Jiang J, Laktabai J, Armstrong J, Holland T, Richards AL, O'Meara WP, 2016. Q fever, scrub typhus, and rickettsial diseases in children, Kenya, 201-2012. Emerg Infect Dis 22: 883-886.

6. Kramme S, An le V, Khoa ND, Trin le V, Tannich E, Rybniker J, Fleischer B, Drosten C, Panning M, 2009. Orientia tsutsugamushi bacteremia and cytokine levels in Vietnamese scrub typhus patients. J Clin Microbiol 47: 586-589.

7. Weitzel T, Aylwin M, Martinez-Valdebenito C, Jiang J, Munita JM, Thompson L, Abarca K, Richards AL, 2018. Imported scrub typhus: first case in South America and review of the literature. Trop Dis Travel Med Vaccines 4: 10.

8. Jensenius M, Montelius R, Berild D, Vene S, 2006. Scrub typhus imported to Scandinavia. Scand J Infect Dis 38: 200-202.

9. Hendershot EF, Sexton DJ, 2009. Scrub typhus and rickettsial diseases in international travelers: a review. Curr Infect Dis Rep 11: $66-72$

10. Vliegenthart-Jongbloed K, de Mendonca Melo M, Slobbe L, Beersma MF, van Genderen PJ, 2013. Imported scrub typhus in The Netherlands. Travel Med Infect Dis 11: 197-199.

11. Suzuki Y, Shimanuki M, Seto J, Yahagi K, Mizuta K, 2016. A case of scrub typhus imported from South Korea to Yamagata, Japan. Jpn J Infect Dis 69: 454-456.

12. Paris DH, Shelite TR, Day NP, Walker DH, 2013. Unresolved problems related to scrub typhus: a seriously neglected lifethreatening disease. Am J Trop Med Hyg 89: 301-307.

13. Thipmontree W, Tantibhedhyangkul W, Silpasakorn S, Wongsawat E, Waywa D, Suputtamongkol Y, 2016. Scrub typhus in northeastern Thailand: eschar distribution, abnormal electrocardiographic findings, and predictors of fatal outcome. Am J Trop Med Hyg 95: 769-773.

14. Kim DM et al., 2007. Distribution of eschars on the body of scrub typhus patients: a prospective study. Am J Trop Med Hyg 76: 806-809.

15. Silpapojakul K, Chupuppakarn S, Yuthasompob S, Varachit B, Chaipak D, Borkerd T, Silpapojakul K, 1991. Scrub and murine typhus in children with obscure fever in the tropics. Pediatr Infect Dis J 10: 200-203.

16. Wei Y, Ma Y, Luo L, Wu X, Huang Y, LiX, Yang Z, 2017. Differences in clinical and laboratory features for different genotypes of Orientia tsutsugamushi in Guangzhou, southern China. Vector Borne Zoonotic Dis 17: 260-267.

17. Kim DM, Kim SW, Choi SH, Yun NR, 2010. Clinical and laboratory findings associated with severe scrub typhus. BMC Infect Dis 10: 108.

18. Yasunaga $\mathrm{H}$, Horiguchi $\mathrm{H}, \mathrm{Kuwabara} \mathrm{K}$, Hashimoto $\mathrm{H}$, Matsuda $\mathrm{S}$, 2011. Delay in tetracycline treatment increases the risk of complications in tsutsugamushi disease: data from the Japanese diagnosis procedure combination database. Intern Med 50: $37-42$.

19. Rajapakse S, Weeratunga P, Sivayoganathan S, Fernando SD, 2017. Clinical manifestations of scrub typhus. Trans $R$ Soc Trop Med Hyg 111: 43-54.

20. Kumar M, Krishnamurthy S, Delhikumar CG, Narayanan P, Biswal N, Srinivasan S, 2012. Scrub typhus in children at a tertiary hospital in southern India: clinical profile and complications. $J$ Infect Public Health 5: 82-88.

21. Narayanasamy DK, Arunagirinathan AK, Kumar RK, Raghavendran VD, 2016. Clinico-laboratory profile of scrub 
typhus-an emerging rickettsiosis in India. Indian J Pediatr 83: 1392-1397.

22. Blacksell SD, Bryant NJ, Paris DH, Doust JA, Sakoda Y, Day NP, 2007. Scrub typhus serologic testing with the indirect immunofluorescence method as a diagnostic gold standard: a lack of consensus leads to a lot of confusion. Clin Infect Dis 44: 391-401.

23. Janardhanan J, Trowbridge $P$, Varghese GM, 2014. Diagnosis of scrub typhus. Expert Rev Anti Infect Ther 12: 1533-1540.

24. Koh GC, Maude RJ, Paris DH, Newton PN, Blacksell SD, 2010. Diagnosis of scrub typhus. Am J Trop Med Hyg 82: 368-370.

25. Kim DM, Kim HL, Park CY, Yang TY, Lee JH, Yang JT, Shim SK, Lee SH, 2006. Clinical usefulness of eschar polymerase chain reaction for the diagnosis of scrub typhus: a prospective study. Clin Infect Dis 43: 1296-1300.

26. Mansueto P, Vitale G, Cascio A, Seidita A, Pepe I, Carroccio A, di Rosa S, Rini GB, Cillari E, Walker DH, 2012. New insight into immunity and immunopathology of rickettsial diseases. Clin Dev Immunol 2012: 967852.

27. Koh Y-S, Yun J-H, Seong S-Y, Choi M-S, Kim I-S, 2004. Chemokine and cytokine production during Orientia tsutsugamushi infection in mice. Microbial Pathogenesis 36: 51-57.

28. Palmer BA, Hetrick FM, Jerrells TJ, 1984. Production of gamma interferon in mice immune to Rickettsia tsutsugamushi. Infect Immun 43: 59-65.

29. Iwasaki H, Mizoguchi J, Takada N, Tai K, Ikegaya S, Ueda T, 2010. Correlation between the concentrations of tumor necrosis factor-alpha and the severity of disease in patients infected with Orientia tsutsugamushi. Int J Infect Dis 14: e328-33.

30. Chung $Y$ et al., 2009. Critical regulation of early Th17 cell differentiation by interleukin-1 signaling. Immunity 30: 576-587.

31. Annunziato F, Cosmi L, Liotta F, Maggi E, Romagnani S, 2009. Human Th17 cells: are they different from murine Th17 cells? Eur J Immunol 39: 637-640.
32. Wei L, Laurence A, Elias KM, O'Shea JJ, 2007. IL-21 is produced by Th17 cells and drives IL-17 production in a STAT3dependent manner. J Biol Chem 282: 34605-34610.

33. Aujla SJ et al., 2008. IL-22 mediates mucosal host defense against gram-negative bacterial pneumonia. Nat Med 14: 275-281.

34. Chen K, McAleer JP, Lin Y, Paterson DL, Zheng M, Alcorn JF, Weaver CT, Kolls JK, 2011. Th17 cells mediate clade-specific, serotype-independent mucosal immunity. Immunity 35: 9971009.

35. Khader SA, Gaffen SL, Kolls JK, 2009. Th17 cells at the crossroads of innate and adaptive immunity against infectious diseases at the mucosa. Mucosal Immunol 2: 403-411.

36. Steinman $L, 2007$. A brief history of $T(H) 17$, the first major revision in the $T(H) 1 / T(H) 2$ hypothesis of T cell-mediated tissue damage. Nat Med 13: 139-145.

37. Yoon HJ, Lee MS, Ki M, Ihm C, Kim D, Kim Y, Yoo SM, 2010. Does IL-17 play a role in hepatic dysfunction of scrub typhus patients? Vector Borne Zoonotic Dis 10: 231-235.

38. Astrup Eet al., 2014. Cytokine network in scrub typhus: high levels of interleukin- 8 are associated with disease severity and mortality. PLoS Negl Trop Dis 8: e2648.

39. Colic M, Gazivoda D, Vucevic D, Vasilijic S, Rudolf R, Lukic A, 2009. Proinflammatory and immunoregulatory mechanisms in periapical lesions. Mol Immunol 47: 101-113.

40. Araujo-Pires AC, Francisconi CF, Biguetti CC, Cavalla F, Aranha AM, Letra A, Trombone AP, Faveri M, Silva RM, Garlet GP, 2014. Simultaneous analysis of Thelper subsets (Th1, Th2, Th9, Th17, Th22, Tfh, Tr1 and Tregs) markers expression in periapical lesions reveals multiple cytokine clusters accountable for lesions activity and inactivity status. J Appl Oral Sci 22: 336-346.

41. Paris DH et al., 2012. Coagulation and inflammation in scrub typhus and murine typhus--a prospective comparative study from Laos. Clin Microbiol Infect 18: 1221-1228. 